\title{
Dobutamine stress for evaluation of right ventricular reserve in pulmonary arterial hypertension
}

\author{
Tripura Sharma ${ }^{1,2,5}$, Edmund M.T. Lau ${ }^{1-3,5}$, Preeti Choudhary ${ }^{1,2}$, Paul J. Torzillo ${ }^{1,3}$, \\ Phillip A. Munoz ${ }^{3}$, Lisa R. Simmons ${ }^{2}$, Robert Naeije ${ }^{4}$ and David S. Celermajer ${ }^{1,2}$ \\ Affiliations: ${ }^{1}$ Discipline of Medicine, Sydney Medical School, University of Sydney, Sydney, Australia. ${ }^{2}$ Dept of \\ Cardiology, Royal Prince Alfred Hospital, Sydney, Australia. ${ }^{3}$ Dept of Respiratory Medicine, Royal Prince Alfred \\ Hospital, Sydney, Australia. ${ }^{4}$ Dept of Pathophysiology, Free University of Brussels, Brussels, Belgium. ${ }^{5}$ Both \\ authors contributed equally.
}

Correspondence: David S. Celermajer, Dept of Cardiology, Royal Prince Alfred Hospital, Camperdown, NSW 2050, Australia. E-mail: david.celermajerdemail.cs.nsw.gov.au

ABSTRACT Right ventricular contractile response to pharmacological stress in pulmonary arterial hypertension $(\mathrm{PAH})$ has not been characterised. We evaluated right ventricular contractile reserve in adults with PAH using dobutamine stress echocardiography.

$16 \mathrm{PAH}$ patients and 18 age-matched controls underwent low-dose dobutamine stress echocardiography. Contractile reserve was assessed by the change ( $\Delta$; peak stress minus rest value) in tricuspid annular plane systolic excursion (TAPSE) and tricuspid annular systolic velocity $\left(\mathrm{S}^{\prime}\right)$. A subgroup of $13 \mathrm{PAH}$ patients underwent treadmill cardiopulmonary exercise testing for peak oxygen uptake $\left(V^{\prime} \mathrm{O}_{2}\right.$ peak).

At rest, TAPSE and $S^{\prime}$ were reduced in the PAH group compared with controls $(1.7 \pm 0.4$ versus $2.4 \pm 0.2 \mathrm{~cm}$ and $9.7 \pm 2.6$ versus $12.5 \pm 1.2 \mathrm{~cm} \cdot \mathrm{s}^{-1}$, respectively; $\left.\mathrm{p}<0.05\right)$. Contractile reserve was markedly attenuated in $\mathrm{PAH}$ compared to controls ( $\triangle$ TAPSE $0.1 \pm 0.2$ versus $0.6 \pm 0.3 \mathrm{~cm}$ and $\Delta \mathrm{S}^{\prime} 4.6 \pm 2.8$ versus $11.2 \pm 3.6 \mathrm{~cm} \cdot \mathrm{s}^{-1}$; $\mathrm{p}<0.0001)$. In the sub-group of $\mathrm{PAH}$ patients with preserved right ventricular systolic function at rest, contractile reserve remained depressed compared to controls. $V^{\prime} \mathrm{O}_{2}$ peak was significantly correlated with $\Delta \mathrm{S}^{\prime}$ $(\mathrm{r}=0.87, \mathrm{p}=0.0003)$ and change in stroke volume $(\mathrm{r}=0.59, \mathrm{p}=0.03)$.

Dobutamine stress can reveal sub-clinical reduction in right ventricular contractile reserve in patients with PAH. A correlation with exercise capacity suggests potential clinical value beyond resting measurements.

@ERSpublications

Dobutamine-induced right ventricular contractile reserve is impaired in PAH and correlates with exercise capacity http://ow.ly/zVg0r

For editorial comments see Eur Respir J 2015; 45: 604-607 [DOI: 10.1183/09031936.00233614].

Received: May 152014 | Accepted after revision: July 092014 | First published online: Oct 302014

Support statement: Funding for this study was received from the National Health and Medical Research Council of Australia Project awarded to D.S. Celermajer (grant no. 1022141), and a National Health and Medical Research Council Post-Graduate Scholarship to E. Lau (no. 633136) and P. Choudhary (no. 1055773).

Conflict of interest: None declared.

Copyright OERS 2015 


\section{Introduction}

Although pulmonary arterial hypertension $(\mathrm{PAH})$ is a disease of the distal pulmonary arteries, the ability of the right ventricle (RV) to adapt to the increased afterload is the main determinant of clinical outcomes in this condition [1]. RV failure is the main cause of death in patients with PAH [2], and most identified prognostic indices have consistently been derived from either direct measurements of RV function or surrogate indicators of RV dysfunction, such as right atrial pressure (RAP) and cardiac output [3-6].

Despite severe elevation of RV afterload in PAH, symptoms of RV failure do not occur at rest until the latter stages of the disease. Thus, it is rational that measurement of RV function under stress may provide incremental value in the clinical assessment of PAH patients. This notion is supported by a recent study where right ventricular contractile reserve was indirectly assessed by the increase in pulmonary artery pressure (PAP) during exercise, and the inability to augment PAP was associated with poorer survival [7].

Inotropic stimulation with dobutamine is an accepted alternative to exercise stress in the evaluation of left ventricular contractile reserve across a range of underlying pathologies including ischaemic heart disease, idiopathic dilated cardiomyopathy and valvular heart disease [8-10]. In contrast, only limited studies have addressed RV contractile reserve and these have predominantly been in the setting of biventricular failure [7, 11-17]. Specifically, assessment of RV contractile reserve using dobutamine stress has not been investigated in PAH. Exercise echocardiography is technically demanding and requires rapid acquisition of images in tachypnoeic patients. Thus, assessment of RV contractile reserve using pharmacological stress is an appealing alternative to exercise stress.

Our study sought to assess RV contractile reserve using low-dose dobutamine stress echocardiography in patients with PAH. We also assessed the association between indices of RV contractile reserve and exercise capacity.

\section{Materials and methods}

A total of 35 subjects participated in the study; 17 subjects with PAH and 18 age-matched healthy controls. The diagnosis of PAH was established according to published guidelines. All PAH patients had invasive confirmation with right heart catheterisation demonstrating mean PAP $\geq 25 \mathrm{mmHg}$, pulmonary artery wedge pressure $\leq 15 \mathrm{mmHg}$ and pulmonary vascular resistance $>3$ Wood units. Healthy controls had no history of cardiorespiratory conditions, no risk factors for pulmonary vascular disease, $<10$ pack-years smoking history and normal baseline transthoracic echocardiography. Pulmonary haemodynamic responses from these study subjects during dobutamine stress have been reported previously [18]. This study was approved by the Local Institutional Ethics Review Committee (Sydney Local Health District, Australia: X10-0260) and all participants provided written informed consent.

\section{Dobutamine stress echocardiography}

Echocardiography images were acquired by either a Vivid 9 system (GE Healthcare, Little Chalfont, UK) or iE33 (Philips Healthcare, Amsterdam, the Netherlands) with a $3.5 \mathrm{MHz}$ transducer by an experienced cardiac sonographer. All patients underwent continuous dobutamine infusion using a low-dose protocol. Dobutamine was increased at increments of $5 \mu \mathrm{g} \cdot \mathrm{kg}^{-1} \cdot \mathrm{min}^{-1}$ at 3 -min intervals up to a maximum of $20 \mu \mathrm{g} \cdot \mathrm{kg}^{-1} \cdot \mathrm{min}^{-1}$. The pre-specified infusion end-points were either reaching the maximum dobutamine dose, heart rate $>120$ beats $\cdot \mathrm{min}^{-1}$ or side-effects requiring cessation. A heart rate of 120 beats $\cdot \mathrm{min}^{-1}$ was chosen for safety reasons to ensure that very high heart rates were not induced in patients with $\mathrm{PAH}$, which could precipitate RV ischaemia. Side-effects requiring cessation of infusion were defined as: 1) hypotension (systolic blood pressure $<85 \mathrm{mmHg}$ ); 2) chest pain; 3) new onset arrhythmias; or 4) intolerable symptoms experienced by subject. All control subjects completed the dobutamine stress echocardiography protocol but one $\mathrm{PAH}$ patient developed self-limiting atrial fibrillation at a dobutamine dose of $10 \mu \mathrm{g} \cdot \mathrm{kg}^{-1} \cdot \mathrm{min}^{-1}$. This patient was excluded from the final data analysis resulting in a final total of 16 assessable PAH patients. No adverse events occurred in any of the other PAH patients.

A standard echocardiogram was performed at baseline. Two-dimensional, M-mode, Doppler echocardiography measurements were performed according to recommendation of the American Society of Echocardiography $[19,20]$. Systolic PAP was measured by peak gradient between the RV and the right atrium from the peak tricuspid regurgitation velocity (TRV) using the Bernoulli equation (systolic PAP = $\left.(\mathrm{TRV})^{2}+\mathrm{RAP}\right)$. RAP was assigned at $5 \mathrm{mmHg}$ for all healthy controls and estimated from the inferior vena cava diameter and collapsibility in PAH subjects. Stroke volume was estimated from the left ventricular outflow tract (LVOT) diameter and LVOT velocity time integral (stroke volume $=$ LVOT velocity time integral $\left.\times \pi(\text { LVOT diameter } / 2)^{2}\right)$. Left ventricular ejection fraction was calculated using Simpson's biplane method. Left atrial and right atrial areas were measured in end-systole from apical four-chamber view. Left ventricular diastolic function was assessed by measuring early (E) and late (A) transmitral flow velocity, and the early diastolic velocity of the medial mitral valve annulus $\left(\mathrm{e}^{\prime}\right)$ in order to derive $\mathrm{E} / \mathrm{A}$ and $\mathrm{E} / \mathrm{e}^{\prime}$ ratios. 
Right ventricular systolic function was assessed at baseline and during dobutamine stress using two established indices of longitudinal contraction: 1) tricuspid annular plane systolic excursion (TAPSE); and 2) pulse tissue Doppler peak tricuspid annular systolic velocity at the lateral annulus $\left(S^{\prime}\right)$. RV contractile reserve was defined as the difference between these values obtained at baseline and peak stress $(\Delta$ TAPSE and $\Delta S^{\prime}$, respectively). At baseline we defined a depressed TAPSE as $<1.6 \mathrm{~cm}$ and $S^{\prime}$ as $<10 \mathrm{~cm} \cdot \mathrm{s}^{-1}$, using reference ranges published in the American Society of Echocardiography guidelines [19]. All values were measured in duplicate for each condition and the average was taken.

\section{Cardiopulmonary exercise testing}

A subgroup of 13 out of 16 patients with $\mathrm{PAH}$ consented to treadmill cardiopulmonary exercise testing (CPET). The remaining three did not provide consent due to lower limb joint disease $(n=1)$ and refusal to participate in exercise testing $(\mathrm{n}=2)$. All CPET were performed within 1 week of dobutamine stress echocardiography on a treadmill using either a Naughton or Modified Bruce protocol according to the subject's estimated exercise capacity. Oxygen uptake $\left(V^{\prime} \mathrm{O}_{2}\right)$, carbon dioxide production and minute ventilation were measured breath-by-breath together with continuous ECG monitoring and blood pressure via cuff sphygmomanometer.

\section{Statistical analysis}

Continuous variables were presented as mean \pm SD, unless otherwise stated. RV systolic function at rest and peak stress were compared using two-way ANOVA with repeated measures, with post hoc Bonferroni correction for multiple comparisons. Spearman's correlation was performed to assess correlation in the $\mathrm{PAH}$ group. A two-sided p-value $<0.05$ was considered statistically significant. Statistical analysis was performed using the Prism 6 package (GraphPad, La Jolla, CA, USA).

\section{Results}

Baseline demographics of the PAH and control groups are shown in table 1. There was a greater proportion of females in the PAH group, but the two groups were otherwise well matched for age and

\begin{tabular}{|c|c|c|c|}
\hline & PAH & Controls & p-value \\
\hline Subjects $\mathrm{n}$ & 16 & 18 & \\
\hline Age years & $56 \pm 9$ & $51 \pm 15$ & 0.25 \\
\hline Male & 25 & 66 & 0.02 \\
\hline Height $\mathrm{cm}$ & $166 \pm 11$ & $168 \pm 11$ & 0.65 \\
\hline Weight kg & $77 \pm 19$ & $75 \pm 15$ & 0.74 \\
\hline Body mass index $\mathrm{kg} \cdot \mathrm{m}^{-2}$ & $27.7 \pm 5.6$ & $26.4 \pm 4.4$ & 0.48 \\
\hline Body surface area $\mathrm{m}^{2}$ & $1.87 \pm 0.28$ & $1.86 \pm 0.23$ & 0.90 \\
\hline \multicolumn{4}{|l|}{ Aetiology } \\
\hline Idiopathic PAH & $6(38)$ & & \\
\hline Scleroderma PAH & $9(56)$ & & \\
\hline Portopulmonary hypertension & $1(16)$ & & \\
\hline \multicolumn{4}{|l|}{ Therapy } \\
\hline Endothelin receptor antagonist & 75 & & \\
\hline Phosphodiesterase- 5 inhibitor & 56 & & \\
\hline Prostanoid & 19 & & \\
\hline Combination therapy & 44 & & \\
\hline NYHA functional class & $2.4 \pm 0.7$ & & \\
\hline I/II/III/IV n & $2 / 6 / 7 / 1$ & & \\
\hline 6MWD m & $410 \pm 149$ & & \\
\hline$V^{\prime} \mathrm{O}_{2}$ peak $\mathrm{mL} \cdot \mathrm{min}^{-1} \cdot \mathrm{kg}^{-1}$ & $16.2 \pm 7.2$ & & \\
\hline \multicolumn{4}{|c|}{ Invasive pulmonary haemodynamics ${ }^{\#}$} \\
\hline Systolic PAP mmHg & $73 \pm 17$ & & \\
\hline Diastolic PAP mmHg & $27 \pm 9$ & & \\
\hline Mean PAP mmHg & $46 \pm 11$ & & \\
\hline PAWP $\mathrm{mmHg}$ & $8 \pm 2$ & & \\
\hline RAP $\mathrm{mmHg}$ & $9 \pm 5$ & & \\
\hline
\end{tabular}

Data are presented as mean $\pm \mathrm{SD}, \%$ or $\mathrm{n}(\%)$, unless otherwise stated. $\mathrm{PAH}$ : pulmonary arterial hypertension; NYHA: New York Heart Association; 6MWD: 6-min walk distance; $V^{\prime} 0_{2}$ peak: peak oxygen uptake; PAP: pulmonary artery pressure; PAWP: pulmonary artery wedge pressure; RAP: right atrial pressure. " : invasive haemodynamics obtained at time of initial diagnostic catheterisation. 
body mass index. The diagnostic categories of PAH included idiopathic PAH $(\mathrm{n}=6)$, scleroderma-associated PAH $(n=9)$ and portopulmonary hypertension $(n=1)$. Baseline disease characteristics encompassed a range of severity as demonstrated by invasive haemodynamics, 6-min walk distance and New York Heart Association (NYHA) functional class. All patients were receiving targeted $\mathrm{PAH}$ therapies at the time of study according to a guideline treatment algorithm [21].

The peak dobutamine dose reached was not significantly different between the two groups (PAH 18.4 \pm 2.9 $\mu \mathrm{g} \cdot \mathrm{kg}^{-1} \cdot \mathrm{min}^{-1}$ versus controls $\left.19.7 \pm 1.2 \mu \mathrm{g} \cdot \mathrm{kg}^{-1} \cdot \mathrm{min}^{-1} ; \mathrm{p}=0.10\right)$. Resting heart rate, as well as peak stress heart rate, were both higher in PAH compared to controls (baseline heart rate: $80 \pm 12$ versus $65 \pm 9$ beats $\cdot \mathrm{min}^{-1}, \mathrm{p}=0.004$; peak heart rate: $113 \pm 17$ versus $96 \pm 12$ beats $\cdot \mathrm{min}^{-1}, \mathrm{p}=0.003$ ). Peak stress resulted in augmentation of systolic PAP from $21 \pm 5$ to $31 \pm 7 \mathrm{mmHg}$ and from $79 \pm 24$ to $108 \pm 30 \mathrm{mmHg}$ in controls and PAH patients, respectively.

\section{Right ventricular contractile reserve}

Baseline echocardiographic parameters are summarised in table 2. Resting RV systolic function was reduced in PAH compared to controls. Resting TAPSE in PAH patients was $1.7 \pm 0.4 \mathrm{~cm}$ and $\mathrm{S}^{\prime}$ was $9.7 \pm 2.6 \mathrm{~cm} \cdot \mathrm{s}^{-1}$. In controls, resting TAPSE and $S^{\prime}$ were both higher at $2.4 \pm 0.2 \mathrm{~cm}$ and $12.5 \pm 1.2 \mathrm{~cm} \cdot \mathrm{s}^{-1}$, respectively (both $\mathrm{p}<0.05$ compared to PAH) (fig. 1). However, 10 (63\%) out of the 16 and seven out of (44\%) the $16 \mathrm{PAH}$ patients had resting TAPSE and $\mathrm{S}^{\prime}$, respectively, within the normal range as defined by TAPSE $\geq 1.6 \mathrm{~cm}$ and $\mathrm{S}^{\prime} \geq 10 \mathrm{~cm} \cdot \mathrm{s}^{-1}[19]$.

RV contractile reserve was markedly reduced in PAH patients compared to controls ( $\triangle$ TAPSE: $0.1 \pm 0.2$ versus $0.6 \pm 0.3 \mathrm{~cm}, \mathrm{p}<0.0001 ; \Delta \mathrm{S}^{\prime}: 4.6 \pm 2.8$ versus $11.2 \pm 3.6 \mathrm{~cm} \cdot \mathrm{s}^{-1}, \mathrm{p}<0.0001$ ) (fig. 2 ). Of the $10 \mathrm{PAH}$ subjects with a preserved TAPSE at rest, $\triangle$ TAPSE was depressed at $0.1 \pm 0.3 \mathrm{~cm} \quad(\mathrm{p}<0.0001$ compared to controls). Similarly, of the seven $\mathrm{PAH}$ patients with a preserved resting $\mathrm{S}^{\prime}, \Delta \mathrm{S}^{\prime}$ was similarly depressed at $5.7 \pm 3.0 \mathrm{~cm} \cdot \mathrm{s}^{-1}(\mathrm{p}=0.0012$ compared to controls).

\section{Relationship between contractile reserve and exercise capacity}

The mean (range) peak $V^{\prime} \mathrm{O}_{2}$ in the PAH group was $16.2 \pm 7.2(9.0-32.9) \mathrm{mL} \cdot \mathrm{kg}^{-1} \cdot \mathrm{min}^{-1}$. There was a significant positive correlation between $\Delta \mathrm{S}^{\prime}$ and peak $V^{\prime} \mathrm{O}_{2}(\mathrm{r}=0.87, \mathrm{p}=0.0003)$ but not between $\Delta$ TAPSE and peak $V^{\prime} \mathrm{O}_{2}(\mathrm{r}=0.21, \mathrm{p}=0.49)$. Change in stroke volume also correlated with exercise capacity $(\mathrm{r}=0.59$, $\mathrm{p}=0.03$ ). When PAH subjects were stratified by NYHA functional class status, those in functional class $1-2$ $(n=8)$ had better contractile reserve compared to those in functional class 3-4 $(n=8)$ when measured by $\Delta \mathrm{S}^{\prime}\left(6.4 \pm 2.5\right.$ versus $\left.2.8 \pm 1.8 \mathrm{~cm} \cdot \mathrm{s}^{-1}, \mathrm{p}=0.005\right)$. Resting TAPSE and $\mathrm{S}^{\prime}$ also correlated significantly with peak $V^{\prime} \mathrm{O}_{2}\left(\mathrm{r}=0.60, \mathrm{p}=0.034\right.$ and $\mathrm{r}=0.62, \mathrm{p}=0.026$, respectively), although less strongly than $\Delta \mathrm{S}^{\prime}$ (fig. 3 ).

\section{Discussion}

To the best of our knowledge this is the first study to assess RV contractile reserve using dobutamine stress echocardiography in patients with PAH. We found that the RV contractile reserve following dobutamine stimulation was markedly reduced in subjects with $\mathrm{PAH}$ and contractile reserve using systolic myocardial velocity at the tricuspid annulus $\left(\Delta S^{\prime}\right)$ correlated significantly with reduced exercise capacity. Furthermore,

\begin{tabular}{|c|c|c|c|}
\hline & PAH & Controls & p-value \\
\hline Subjects $\mathrm{n}$ & 16 & 18 & \\
\hline \multicolumn{4}{|l|}{ Left heart parameters } \\
\hline LVEF \% & $71 \pm 8$ & $64 \pm 6$ & 0.005 \\
\hline Left atrial area $\mathrm{cm}^{2}$ & $16 \pm 3$ & $13 \pm 3$ & 0.003 \\
\hline$E / e^{\prime}$ & $12.3 \pm 4.7$ & $8.8 \pm 2.8$ & 0.013 \\
\hline E/A ratio & $1.0 \pm 0.2$ & $1.3 \pm 0.5$ & 0.008 \\
\hline \multicolumn{4}{|l|}{ Right heart parameters } \\
\hline Right atrial area $\mathrm{cm}^{2}$ & $23 \pm 7$ & $14 \pm 3$ & $<0.001$ \\
\hline Systolic PAP & $79 \pm 24$ & $21 \pm 5$ & $<0.001$ \\
\hline TAPSE cm & $1.7 \pm 0.4$ & $2.4 \pm 0.2$ & $<0.001$ \\
\hline $\mathrm{S}^{\prime} \mathrm{cm} \cdot \mathrm{s}^{-1}$ & $9.7 \pm 2.6$ & $12.5 \pm 1.2$ & $<0.001$ \\
\hline
\end{tabular}

Data are presented as mean \pm SD, unless otherwise stated. PAH: pulmonary arterial hypertension; LVEF: left ventricular ejection fraction; $E / e^{\prime}$ : early $(E)$ to early diastolic mitral annular velocity $\left(e^{\prime}\right)$; $E / A$ : early $(E)$ to late (A) ventricular filling velocities; PAP: pulmonary artery pressure; TAPSE: tricuspid annular plane systolic excursion; S': tricuspid annulus systolic myocardial velocity. 
a)
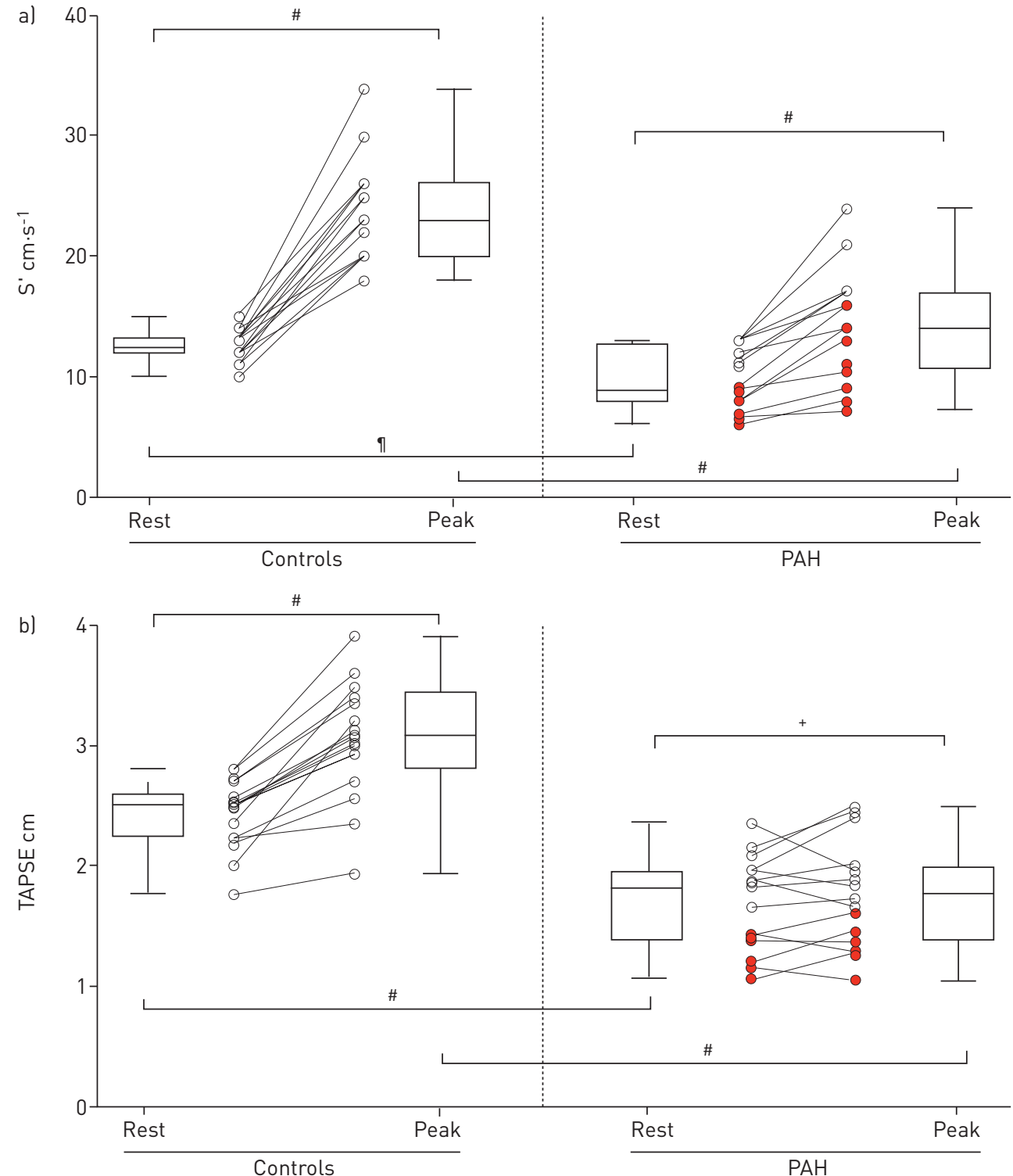

FIGURE 1 Right ventricular systolic function at rest and during peak dobutamine stress. In controls, dobutamine stress led to significant augmentations in both a) tricuspid annulus systolic myocardial velocity $\left(\mathrm{S}^{\prime}\right)$ and $\mathrm{b}$ ) tricuspid annular plane systolic excursion (TAPSE) $\left(12.5 \pm 1.2\right.$ to $23.7 \pm 3.9 \mathrm{~cm} \cdot \mathrm{s}^{-1}$ and $2.4 \pm 0.2$ to $3.1 \pm 0.5 \mathrm{~cm}$, respectively). In pulmonary arterial hypertension (PAH) patients, only $\mathrm{S}^{\prime}$ increased during dobutamine stress $\left(9.7 \pm 2.6\right.$ to $\left.14.3 \pm 4.6 \mathrm{~cm} \cdot \mathrm{s}^{-1}\right)$ but no significant augmentation in TAPSE was observed $(1.7 \pm 0.4$ to $1.8 \pm 0.4 \mathrm{~cm})$. Subgroups of PAH subjects with depressed TAPSE and $\mathrm{S}^{\prime}$ values at rest are indicated in red. ${ }^{*}: \mathrm{p}<0.0001 ;{ }^{9}: \mathrm{p}=0.034{ }^{+}: \mathrm{p}=0.69$.

we found that even in $\mathrm{PAH}$ patients where RV parameters were within the "normal range" at baseline, contractile reserve remained significantly depressed compared to healthy controls. This suggests that the use of dobutamine stress may potentially unmask latent RV dysfunction that is not apparent at rest. A significant correlation between indices of RV contractile reserve and exercise capacity in PAH patients supports the potential clinical utility of dobutamine stress echocardiography, given that exercise capacity is a powerful predictor of prognosis in this condition [22].

Due to the complex geometry of the RV, accurate volumetric assessment with two-dimensional echocardiography is difficult. Even with three-dimensional echocardiography, volumetrics can be inaccurate [23]. Magnetic resonance imaging is the gold standard for assessing RV volumes but its expense limits its current role in the routine bedside assessment of RV function, and exercise magnetic resonance imaging for RV function is currently not well established. Due to the unique myocardial fibre orientation of the RV, base-to-apex longitudinal contraction is considered to be particularly important in RV emptying $[24,25]$. TAPSE and $S^{\prime}$ represent two of the most commonly used, reproducible and validated indices of RV systolic function in clinical practice [19]. Furthermore, measures of longitudinal RV systolic 

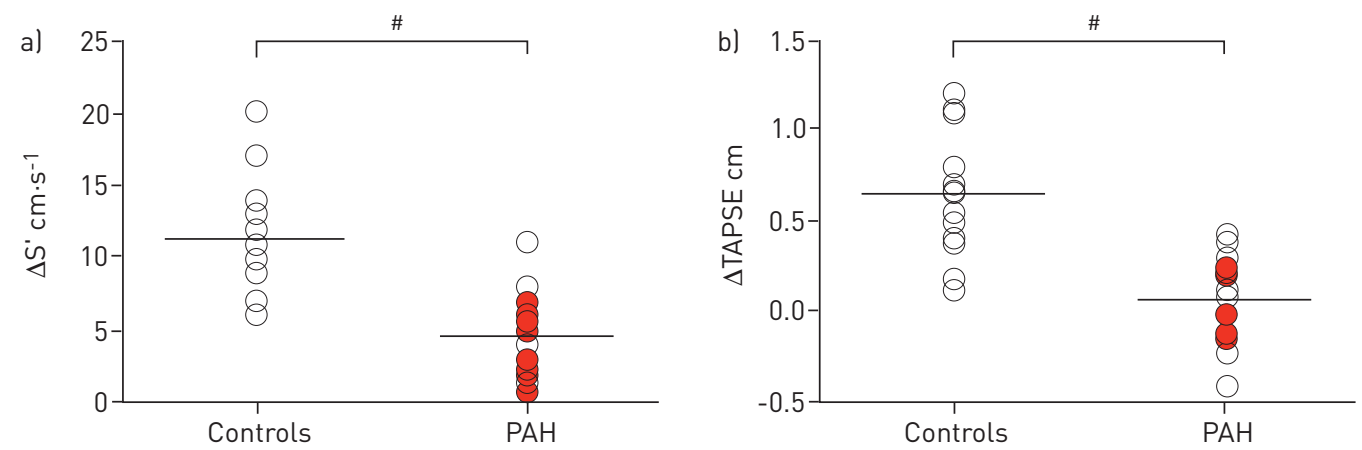

FIGURE 2 Right ventricular contractile reserve in controls versus pulmonary arterial hypertension (PAH) patients. Both change $(\Delta)$ in a) tricuspid annulus systolic myocardial velocity $\left(S^{\prime}\right)$ and b) tricuspid annular plane systolic excursion (TAPSE) were markedly higher in controls compared to PAH $\left(\Delta \mathrm{S}^{\prime}: 11.2 \pm 3.6\right.$ versus $4.6 \pm 2.8 \mathrm{~cm} \cdot \mathrm{s}^{-1}, \Delta$ TAPSE: $0.6 \pm 0.3$ versus $0.1 \pm 0.2 \mathrm{~cm}$ ). Subgroups of PAH subjects with depressed TAPSE and $\mathrm{S}^{\prime}$ values at rest are indicated in red. ${ }^{*}$ : $\mathrm{p}<0.0001$.

function have been demonstrated to predict prognosis in $\mathrm{PAH}[3,26]$. For this reason, we elected to study these two particular RV indices for the present study, for both simplicity and potential clinical application.

The assessment of left ventricular contractile reserve using stress echocardiography is a well-established technique for left heart disease [27]. The incremental prognostic value of left ventricular contractile reserve over resting measurements has been demonstrated for ischaemic heart disease, valvular heart disease and idiopathic dilated cardiomyopathy [13, 28, 29]. Only two previous studies have specifically evaluated RV contractile reserve in PAH and employed exercise Doppler echocardiography protocols [7, 17]. In the study by GRUNIG et al. [7], RV contractile reserve was assessed indirectly by the augmentation of PAP during exercise, rather than by direct measurements of RV function. PAH patients who were able to augment PAP above the median value of $30 \mathrm{mmHg}$ had a significantly improved prognosis on follow-up. The physiological basis of exercised-induced PAP augmentation as a surrogate parameter of contractile reserve is based on the premise that any increase in PAP during exercise results from an increase in
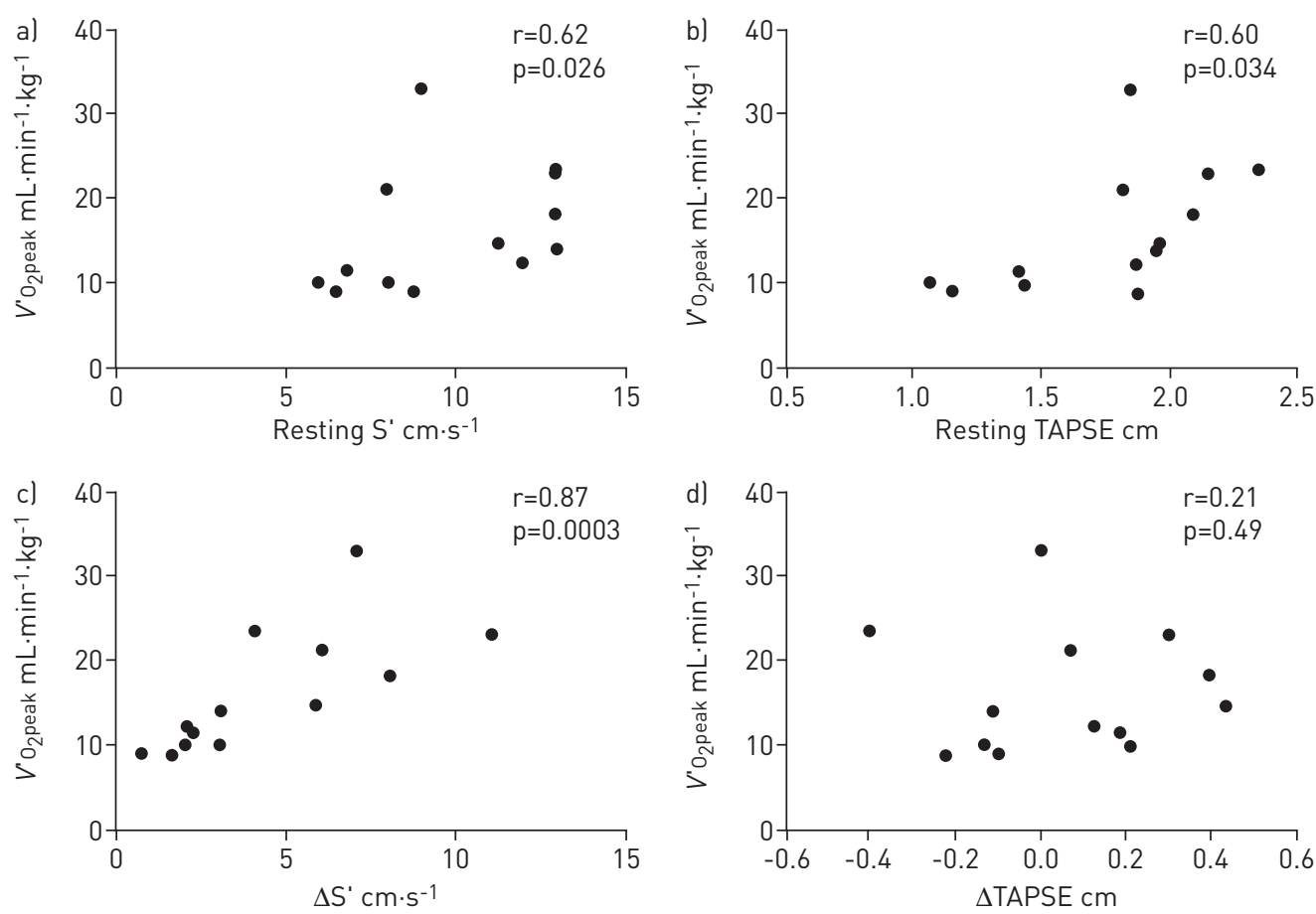

FIGURE 3 Correlation between a, b) resting right ventricular systolic function and $c, d)$ contractile reserve with peak oxygen uptake $\left(V^{\prime} \mathrm{O}_{2}\right.$ peak). The best correlate of $V^{\prime} \mathrm{O}_{2}$ peak was change $(\Delta)$ in tricuspid annulus systolic myocardial velocity $\left(\mathrm{S}^{\prime}\right)$. Resting $\mathrm{S}^{\prime}$ and tricuspid annular plane systolic excursion (TAPSE) also showed moderate correlation with $V^{\prime} \mathrm{O}_{2}$ peak but no relationship was found with $\triangle$ TAPSE. 
cardiac output, which in turn is a reflection of the ability of the RV to increase its stroke volume under stress. In another study, AlmeIDA et al. [17] made direct measurements of RV systolic function during treadmill exercise. They found $\Delta S^{\prime}$ and $\Delta$ TAPSE augmentations in PAH patients of $4.3 \pm 3.3 \mathrm{~cm} \cdot \mathrm{s}^{-1}$ and $0.06 \pm 0.25 \mathrm{~cm}$, respectively; values very similar to those disclosed by dobutamine stress in our study.

The use of low-dose dobutamine stress echocardiography is potentially a simpler and more appealing modality for the bedside assessment of RV contractile reserve, compared to exercise stress. Exercise echocardiography is technically challenging for the RV and particularly so in the breathless patient due to respiratory motion and the need for rapid image acquisition during exercise. With dobutamine stress echocardiography we were able to obtain, with sufficient quality, direct measurements of RV function in all subjects at peak stress, rather than rely on a surrogate marker such as exercise-induced PAP augmentation. Furthermore, direct measurement of RV function is likely to be preferable, as PAP augmentation during exercise is not only on dependent RV stroke volume reserve, but also on the individual patient's chronotropic response and the slope of the pressure-flow relationship of the pulmonary circulation during exercise [30].

It was of interest that we found a significant correlation between contractile reserve measured by $\Delta \mathrm{S}^{\prime}$ and peak $V^{\prime} \mathrm{O}_{2}$. Even though patients with $\mathrm{PAH}$ may present with mild-to-moderate ventilation/perfusion mismatch abnormalities, the main determinant of exercise capacity is right ventricular function (and cardiac output) [31]. In PAH, exercise capacity is dependent on the ability of the RV to respond to the increase in cardiac output required for the metabolic requirements of exercise [32]. Thus, it is perceivable that measures of RV contractile reserve may, therefore, be coupled with exercise capacity. The lack of correlation between $\triangle$ TAPSE with exercise capacity may be due to a number of factors. Although TAPSE and $S^{\prime}$ both represent measures of longitudinal contraction at the tricuspid annulus, they are fundamentally different in that TAPSE measures the distance and $S^{\prime}$ measures the peak velocity of systolic contraction. For this reason, it is likely that $S^{\prime}$ may be more sensitive to the inotropic effects of dobutamine and, thus, may better reflect augmentation of RV stroke volume compared to TAPSE. Furthermore, studies in healthy subjects indicate that exercise stress augments TAPSE by $\sim 20 \%$ relative to baseline $(\sim 0.5 \mathrm{~cm})$ but $\mathrm{S}^{\prime}$ increases by $\sim 80 \%\left(\sim 10 \mathrm{~cm} \cdot \mathrm{s}^{-1}\right)$ [17]. Thus, the relatively small augmentation in TAPSE (even in normal subjects) suggests that assessment of RV reserve using TAPSE may suffer from larger noise-to signal ratio compared to $S^{\prime}$. In fact, our study found that TAPSE did not change significantly following dobutamine stress in PAH patients, which is concordant with the findings of the recent exercise study by Almeida et al. [17].

\section{Limitations}

The major limitation of our study was a relatively small number of subjects. However, this was an exploratory study to determine the feasibility, safety and potential utility of low dose dobutamine stress echocardiography for the assessment of RV contractive reserve in PAH and had sufficient power for clear separation of groups. We acknowledge that both TAPSE and $S^{\prime}$ have drawbacks as measurements of RV function, as ideal measures of ventricular contractility should be load independent, such as end-systolic ventricular elastance derived from invasively measured pressure-volume loops. Nevertheless, longitudinal measures of RV systolic function are simple and reproducible parameters that are utilised widely in clinical practice and, at present, there remains no ideal load-independent measurement of RV function using two-dimensional echocardiography. It remains to be determined whether such longitudinal measures of RV systolic function are sufficient to characterise RV contractile reserve.

We did not assess contractile reserve using other RV parameters, such as fractional area change, Tei-index or tricuspid annulus isovolumic acceleration. Our own experience and that of other authors has found fractional area change to suffer from large interobserver variations [33], often the result of difficulty in clearly defining endocardial borders due to RV trabeculations. Furthermore, accurate measurement of RV area is limited by the complex geometry of this chamber. Both Tei-index and isovolumic acceleration have large variability depending on heart rate and, thus, we avoided these parameters due to the chronotropic effects of dobutamine (as would also apply to exercise).

Our dobutamine stress echocardiography protocol represents a modification of the protocol used for assessment of myocardial ischaemia, which conventionally employs dobutamine doses up to 40 $\mu \mathrm{g} \cdot \mathrm{kg}^{-1} \cdot \mathrm{min}^{-1}$ with or without additional atropine [34]. Patients with severe PAH may not tolerate extreme heart rates and induction of arrhythmias is also of particular concern in this study. Our protocol, with a peak dose of $20 \mu \mathrm{g} \cdot \mathrm{kg}^{-1} \cdot \mathrm{min}^{-1}$ and limiting heart rate to a maximum of $120 \mathrm{beats} \cdot \mathrm{min}^{-1}$, appeared generally safe and well tolerated. It has been demonstrated that a heart rate of around $\sim 120$ beats $\cdot \mathrm{min}^{-1}$ is also close to the maximum response in severe PAH patients because of decreased chronotropic response [35]. The optimal dosing protocol of dobutamine stress for the assessment of RV contractile reserve in PAH requires further clarification. 
Not all PAH patients underwent cardiopulmonary exercise testing, which could have had an influence on our overall results concerning the relationship between RV contractile reserve and exercise capacity. Finally, larger prospective studies are required to determine the clinical relevance of different echocardiography derived parameters of RV contractile reserve, the limits of normal contractile reserve in healthy individuals, and whether threshold values of RV contractile reserve can be predictive of poorer outcomes in PAH.

\section{Conclusions}

We demonstrate that patients with $\mathrm{PAH}$ exhibit markedly diminished RV contractile reserve during dobutamine stress echocardiography, and abnormal stress responses occur even in those with relatively preserved resting RV systolic function. RV contractile reserve correlates with exercise capacity, suggesting the potential clinical value of measures of RV contractile reserve beyond resting measurements.

\section{References}

1 Vonk-Noordegraaf A, Haddad F, Chin KM, et al. Right heart adaptation to pulmonary arterial hypertension: physiology and pathobiology. J Am Coll Cardiol 2013; 62 Suppl. 25:D22-D33.

2 Tonelli AR, Arelli V, Minai OA, et al. Causes and circumstances of death in pulmonary arterial hypertension. Am J Respir Crit Care Med 2013; 188: 365-369.

3 Forfia PR, Fisher MR, Mathai SC, et al. Tricuspid annular displacement predicts survival in pulmonary hypertension. Am J Respir Crit Care Med 2006; 174: 1034-1041.

4 van Wolferen SA, Marcus JT, Boonstra A, et al. Prognostic value of right ventricular mass, volume, and function in idiopathic pulmonary arterial hypertension. Eur Heart J 2007; 28: 1250-1257.

5 D'Alonzo GE, Barst RJ, Ayres SM, et al. Survival in patients with primary pulmonary hypertension. Results from a national prospective registry. Ann Intern Med 1991; 115: 343-349.

6 Humbert M, Sitbon O, Chaouat A, et al. Survival in patients with idiopathic, familial, and anorexigen-associated pulmonary arterial hypertension in the modern management era. Circulation 2010; 122: 156-163.

7 Grunig E, Tiede H, Enyimayew EO, et al. Assessment and prognostic relevance of right ventricular contractile reserve in patients with severe pulmonary hypertension. Circulation 2013; 128: 2005-2015.

8 Eichhorn EJ, Grayburn PA, Mayer SA, et al. Myocardial contractile reserve by dobutamine stress echocardiography predicts improvement in ejection fraction with $\beta$-blockade in patients with heart failure. Circulation 2003; 108: 2336-2341.

9 Naqvi TZ, Goel RK, Forrester JS, et al. Myocardial contractile reserve on dobutamine echocardiography predicts late spontaneous improvement in cardiac function in patients with recent onset idiopathic dilated cardiomyopathy. J Am Coll Cardiol 1999; 34: 1537-1544.

10 Pinamonti B, Perkan A, Di Lenarda A, et al. Dobutamine echocardiography in idiopathic dilated cardiomyopathy: clinical and prognostic implications. Eur J Heart Fail 2002; 4: 49-61.

11 Sade LE, Ozin B, Ulus $\mathrm{T}$, et al. Right ventricular contractile reserve in mitral stenosis: implications on hemodynamic burden and clinical outcome. Int J Cardiol 2009; 135: 193-201.

12 Gorcsan J 3rd, Murali S, Counihan PJ, et al. Right ventricular performance and contractile reserve in patients with severe heart failure. Assessment by pressure-area relations and association with outcome. Circulation 1996; 94: 3190-3197.

13 Otasevic P, Popovic Z, Pratali L, et al. Right $v s$. left ventricular contractile reserve in one-year prognosis of patients with idiopathic dilated cardiomyopathy: assessment by dobutamine stress echocardiography. Eur J Echocardiogr 2005; 6: 429-434.

14 Coma-Canella I, del Val Gomez Martinez M, Terol I, et al. Radionuclide assessment of right ventricular contractile reserve after acute myocardial infarction. Am J Cardiol 1994; 74: 982-986.

15 Ishii H, Harada K, Toyono M, et al. Usefulness of exercise-induced changes in plasma levels of brain natriuretic peptide in predicting right ventricular contractile reserve after repair of tetralogy of Fallot. Am J Cardiol 2005; 95: 1338-1343.

16 La Gerche A, Burns AT, D’Hooge J, et al. Exercise strain rate imaging demonstrates normal right ventricular contractile reserve and clarifies ambiguous resting measures in endurance athletes. J Am Soc Echocardiogr 2012; 25: $253-262$.

17 Almeida AR, Loureiro MJ, Lopes L, et al. Echocardiographic assessment of right ventricular contractile reserve in patients with pulmonary hypertension. Rev Port Cardiol 2014; 33: 155-163.

18 Lau EM, Vanderpool RR, Choudhary P, et al. Dobutamine stress echocardiography for the assessment of pressure-flow relationships of the pulmonary circulation. Chest 2014; 146: 959-966.

19 Rudski LG, Lai WW, Afilalo J, et al. Guidelines for the echocardiographic assessment of the right heart in adults: a report from the American Society of Echocardiography endorsed by the European Association of Echocardiography, a registered branch of the European Society of Cardiology, and the Canadian Society of Echocardiography. J Am Soc Echocardiogr 2010; 23: 685-713.

20 Quinones MA, Otto CM, Stoddard M, et al. Recommendations for quantification of Doppler echocardiography: a report from the Doppler Quantification Task Force of the Nomenclature and Standards Committee of the American Society of Echocardiography. J Am Soc Echocardiogr 2002; 15: 167-184.

21 Galie N, Hoeper MM, Humbert M, et al. Guidelines for the diagnosis and treatment of pulmonary hypertension: the Task Force for the Diagnosis and Treatment of Pulmonary Hypertension of the European Society of Cardiology (ESC) and the European Respiratory Society (ERS), endorsed by the International Society of Heart and Lung Transplantation (ISHLT). Eur Heart J 2009; 30: 2493-2537.

22 Miyamoto S, Nagaya N, Satoh T, et al. Clinical correlates and prognostic significance of six-minute walk test in patients with primary pulmonary hypertension. Comparison with cardiopulmonary exercise testing. Am J Respir Crit Care Med 2000; 161: 487-492. 
23 Shimada YJ, Shiota M, Siegel RJ, et al. Accuracy of right ventricular volumes and function determined by three-dimensional echocardiography in comparison with magnetic resonance imaging: a meta-analysis study. J Am Soc Echocardiogr 2010: 943-953.

24 Brown SB, Raina A, Katz D, et al. Longitudinal shortening accounts for the majority of right ventricular contraction and improves after pulmonary vasodilator therapy in normal subjects and patients with pulmonary arterial hypertension. Chest 2011; 140: 27-33.

25 Naito H, Arisawa J, Harada K, et al. Assessment of right ventricular regional contraction and comparison with the left ventricle in normal humans: a cine magnetic resonance study with presaturation myocardial tagging. $\mathrm{Br} \mathrm{Heart}$ J 1995; 74: 186-191.

26 Guazzi M, Bandera F, Pelissero G, et al. Tricuspid annular plane systolic excursion and pulmonary arterial systolic pressure relationship in heart failure: an index of right ventricular contractile function and prognosis. Am J Physiol Heart Circ Physiol 2013; 305: H1373-H1381.

27 Francis GS, Desai MY. Contractile reserve: are we beginning to understand it?. JACC Cardiovasc Imaging 2008; 1 : 727-728.

28 Chaudhry FA, Tauke JT, Alessandrini RS, et al. Prognostic implications of myocardial contractile reserve in patients with coronary artery disease and left ventricular dysfunction. J Am Coll Cardiol 1999; 34: 730-738.

29 Magne J, Mahjoub H, Dulgheru R, et al. Left ventricular contractile reserve in asymptomatic primary mitral regurgitation. Eur Heart J 2014; 35: 1608-1616.

30 Naeije R, Chesler N. Pulmonary circulation at exercise. Compr Physiol 2012; 2: 711-741.

31 Naeije R. Treatment of right heart failure on pulmonary arterial hypertension: is going left a step in the right direction?. Eur Respir Rev 2010; 19: 4-6.

32 Blumberg FC, Arzt M, Lange T, et al. Impact of right ventricular reserve on exercise capacity and survival in patients with pulmonary hypertension. Eur J Heart Fail 2013; 15: 771-775.

33 Pavlicek M, Wahl A, Rutz T, et al. Right ventricular systolic function assessment: rank of echocardiographic methods vs. cardiac magnetic resonance imaging. Eur J Echocardiogr 2011; 12: 871-880.

34 Sicari R, Nihoyannopoulos P, Evangelista A, et al. Stress echocardiography expert consensus statement: European Association of Echocardiography. Eur J Echocardiogr 2008; 9: 415-437.

35 Deboeck G, Niset G, Lamotte M, et al. Exercise testing in pulmonary arterial hypertension and in chronic heart failure. Eur Respir J 2004; 23: 747-751. 